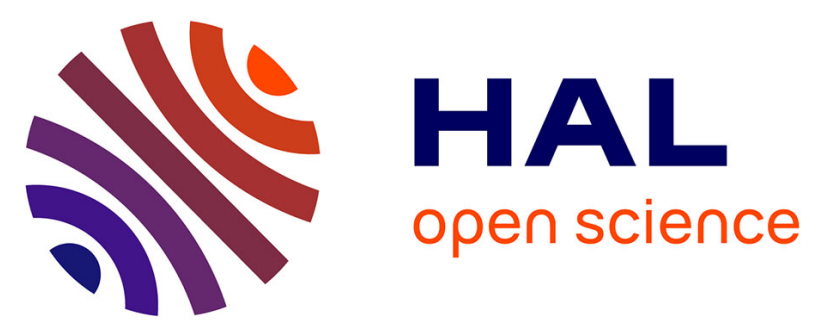

\title{
A Generic Ontology-Based Information Model for Better Management of Microgrids
}

Khouloud Salameh, Richard Chbeir, Haritza Camblong, Gilbert Tekli, Ionel Vechiu

\section{- To cite this version:}

Khouloud Salameh, Richard Chbeir, Haritza Camblong, Gilbert Tekli, Ionel Vechiu. A Generic Ontology-Based Information Model for Better Management of Microgrids. 11th IFIP International Conference on Artificial Intelligence Applications and Innovations (AIAI 2015), Sep 2015, Bayonne, France. pp.451-466, 10.1007/978-3-319-23868-5_33 . hal-01254493

\section{HAL Id: hal-01254493 https://hal.science/hal-01254493}

Submitted on 21 Oct 2016

HAL is a multi-disciplinary open access archive for the deposit and dissemination of scientific research documents, whether they are published or not. The documents may come from teaching and research institutions in France or abroad, or from public or private research centers.
L'archive ouverte pluridisciplinaire HAL, est destinée au dépôt et à la diffusion de documents scientifiques de niveau recherche, publiés ou non, émanant des établissements d'enseignement et de recherche français ou étrangers, des laboratoires publics ou privés.

\section{(c)(1)}

Distributed under a Creative Commons Attribution| 4.0 International License 


\title{
A Generic Ontology-Based Information Model for Better Management of Microgrids
}

\author{
Khouloud Salameh ${ }^{1}$, Richard Chbeir $^{1}$, Haritza Camblong ${ }^{2}$, \\ Gilbert Tekli ${ }^{1}$, Ionel Vechiu ${ }^{3}$ \\ ${ }^{1}$ LIUPPA Laboratory, University of Pau and Adour Countries (UPPA) \\ 64600 Anglet, France \\ \{khouloud.salameh, richard.chbeir, gilbert.tekli\}@univ-pau.fr \\ ${ }^{2}$ Departement of Systems Engineering and Control \\ University of Basque Country (UPV-EHU) \\ Europa Plaza 1, Donostia20018, Spain \\ aritza.camblong@ehu.es \\ ${ }^{3}$ ESTIA Research, Technopôle Izarbel \\ 64210 Bidart, France, \\ i.vechiu@estia.fr
}

\begin{abstract}
In this "renewable era", researchers' eyes are diverted to the Microgrids (emerging power systems consisting of a number of heterogeneous components including renewable and non-renewable energy sources, storage systems and energy loads) to exploit their functionalities in order to improve today's power systems. This work addresses two significant issues encountered in the Microgrid (MG) management systems: 1) the semantic interoperability, highlighting the need to ensure a seamless communication between several heterogeneous components, and 2) the multi-objective aspect of the $\mathrm{MG}$ emphasizing the need to model all the aspects involved in the achievement of the MG objectives. Here, we propose OntoMG, a full-fledged Microgrid ontology, to resolve these two issues by providing an interoperable information model with existing standards and by modeling all the aspects/functionalities involved in the achievement of the MG objectives, namely: Identification, Operational, Mobility, Economical and Ecological. This research is undertaken in collaboration with Jema Irizar Group, leader of the ISare Microgrid Project, a real-size MG built in the Basque Country.
\end{abstract}

Keywords: Microgrids, Interoperability, Multi-objective aspect, Ontology-based information system

\section{Introduction}

With the advancements of "smart" technologies and equipment, as well as the growing need for reliable ecological energy supplies [1], new services and opportunities are emerging in the electricity domain. Here comes the "Microgrids" [3], a new paradigm for the operation of power systems (cf. Fig. 1). To "keep the lights on", they are designed to provide reliable power by incorporating local generation units/systems 
based on renewable and conventional power sources, energy storage systems and energy consumption loads. Moreover, MGs are supposed to play an essential role in power supply systems, by enhancing efficient power distribution and cost effectiveness (manifested by the installation of power sources nearby the consumers' loads).

MGs are adopted more and more in different countries and locations [4]. However, as it is the case with most of new technologies, several needs and challenges have emerged. One major challenge is related to provide appropriate "interoperability", since an MG consists of a number of heterogeneous connected components, built and supplied by different organizations with diversified targets and ensuing different protocols [5]. This underlines the need to ensure a seamless information exchange between its components.

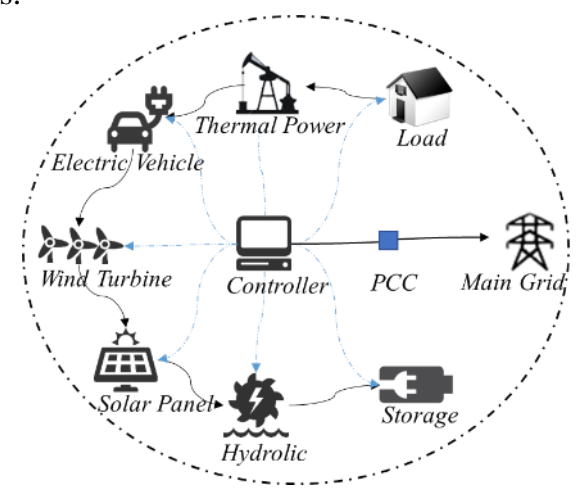

Fig. 1. Microgrid Architecture Example

In this work, an MG and its interoperability are observed within three layers as discussed in [9] (cf. Fig. 2): Field Layer, Information/Knowledge Layer, and Management Layer. More details about the layers will be provided in Section 3.

Another important challenge is related to defining an MG model that takes into account all the components aspects involved in the accomplishment of the MG's objectives besides optimizing network operations (operational aspect), since an MG can be seen as a multi-objective system that relies on a considerable interaction among different stakeholders (i.e., energy sources, energy consumption loads, etc.), having each its objectives.

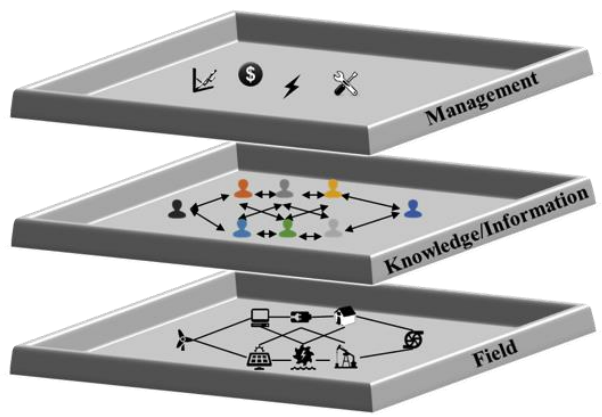

Fig. 2. Microgrid Architecture Layers 
To do so, we identify five aspects, each related to a category of objectives:

- The identification aspect (Id) consists of associating a unique identity for each stakeholder enabling an easier component recognition and implicit information extraction

- The operation aspect (Op) aims at optimizing the network operations (e.g., minimizing power losses, voltage variations, device loading, etc.)

- The mobility aspect (Mob) captures the components moves during its lifetime, in order to optimize the MG cost efficiency and emphasize the stakeholder's adaptability

- The economical aspect (Eco) aims at minimizing total costs while considering the components' participation in the Energy Market

- The ecological aspect (Ecolo) related to the component's participation in the environment, dispatches the MG units with lower specific emissions levels with higher priority, disregarding economic and operational aspects

The goal of this research is to address the above issues and challenges. Our contribution consists of defining an ontology-based Microgrid model, OntoMG, capable of: mapping with several existing MG information models, addressing the basic interoperability between layers, as well as solving the multi-objective aspects of the MG. An illustration of the use of OntoMG will be done through ISare $\mathrm{MG}^{1}$, a real MG built is the Basque Country.

The rest of the paper is organized as follows. Section 2 presents the state of the art of existing power systems information models. Section 3 describes our OntoMG ontology through its main concepts. In Section 4, we show how ISare MG is represented using our ontology. Section 4 concludes the paper and discusses future directions.

\section{State of the art}

Several approaches have been provided in the literature addressing the problem of "Power system information modeling". They can be categorized into ontology-based and non-ontology-based approaches as detailed in what follows.

\subsection{Ontology Based Approaches}

\subsubsection{Belgrade Ontology}

In [10], the authors developed an ontology aiming to provide a classification for the MGs by providing a representation of its components and their control parameters. Complying with the Suggested Upper Merged Ontology ${ }^{2}$, the proposed ontology aims to classify the MG domain in two basic concepts: the Physical and the Abstract concepts. The Physical concept serves for describing the hardware components of the Microgrid (i.e., production unit, storage unit, etc.) with a set of related properties. Concerning the Abstract concept, two subclasses are introduced: i) Management class,

\footnotetext{
${ }^{1}$ This work is done in collaboration with Jema Irizar Group, leader of the ISare project.

${ }^{2} \mathrm{http}: / /$ www.adampease.org/OP/
} 
containing information related to the energy trading and ii) Policy class, defining specific rules related to the MG operations.

\subsubsection{Prosumer Ontology}

In [11], the authors work on the classification of the MG components using several predefined use cases. Based on the UK property classification ${ }^{3}$, five power consumption patterns are identified, namely: 1) "commercial premises", 2) "business related premises", 3) "residential premises", 4) "non-residential", and 5) "industrial premises". Concerning the energy sources classification, two categories were also introduced in [11]: "renewable" and "non-renewable" energy sources, while three energy storage systems categories are identified, according to the type, produced power and charge and discharge efficiency, namely: 1) "energy management", 2) "power quality", and 3) "bridging power". And finally, the "component connectivity" focuses on enabling the exact connectivity relationships between the producers and the consumers. Besides the components classification, a class "events" has been added to consider the Complex Event Processing [12].

\subsubsection{Upper Ontology for power engineering application}

Based on the Common Information Model (CIM) [13] (c.f. Section 2.2.1 for more details), the authors in [14] propose an ontology that mainly aims at monitoring the health status of the power systems. The main concepts of this ontology are power system's components, measurements, data interpretations, and components' operations in the system. This model supports the exchange of messages between agents, even though not explicitly defined. Despite the adoption of this model by several applications, CIM usually needs to be enriched with slightly additional concepts to cover all the required information.

\subsection{Non Ontology Based Models}

\subsubsection{Common Information Model}

The Common Information Model (CIM) [13] is a widely accepted energy information model being part of the IEC 61970 standards. Its main objective is to develop a platform independent data model for enabling better smart grid interoperability. This model includes the exchange of information between market participants and market operators as well as communication between market operators. Yet, the CIM cannot fully describe the MG itself nor its devices. In [11], an extension of CIM is provided, aiming to model the Microgrid components with unified modeling language (UML) while integrating different branch models for solar generation, wind power, energy storage equipment, etc. Our work will be based on this approach since it is one of the most adopted conceptual models with concrete implementation used to describe power system information.

\footnotetext{
${ }^{3}$ http://www.pearl-coutts.co.uk/resources/property-articles/uk-property-classifications
} 


\subsubsection{MIRABEL FlexEnergy Data Model}

The MIRABEL smart grid system comes to model the flexibility in energy demand and supply that incorporate the "power profile" concept which associates a consumption/production schedule for each device. In order to achieve such flexibility in energy demand and supply in the power grid, a data model has been developed in [15] consisting of five main classes: device, actor, energy profile, constraint and flexoffer. A device is an energy consumer or producer that has a specific energy load over a certain time span. An actor has minimum or maximum demands on their energy load, price and time. These constraints are issued toward the devices owned by the actor. The flex-offer class defines a demand mechanism consisting of two types of demands: flexible demand and non-flexible demand. Flexible demand can often be shifted from the peak demand times to lower demand times, while non-flexible demand should be satisfied immediately with a minimum time delay.

\subsubsection{OASIS Energy Interoperability}

OASIS Energy Interoperation [16] enables collaborative use of energy in a power network. It defines XML-based vocabularies for the interoperable exchange of information related to energy prices and bids (demand and response), emergency signals and the prediction of loads consumption. This information relies on the "WSCalendar" [17] and "EMIX specification" [18] . The first defines how to specify and communicate the duration and time of a schedule, while the later specifies the semantics (i.e., definitions of price and products) in energy markets.

\subsubsection{Facility Smart Grid Information Standard}

The Facility Smart Grid Information Standard (NIST) [19] is developed in the aim of enabling energy consuming devices and control systems in the consumers bounds so to manage electrical loads and energy sources ensuring an ease communication with the smart grid. To achieve this, an object-oriented information model is defined to support a wide range of energy management applications and electrical service provider interactions. Their proposed information model provides a standard to describe and manage information on combined energy loads consumption and predictions.

\subsection{Discussion}

In this section, a comparison between the existing approaches is presented, highlighting their strengths and drawbacks with respect to their ability to resolve the interoperability issue in the MG, and the integration of the necessary aspects allowing the achievement of the MG objectives.

\subsubsection{Interoperability aspect}

Table 1 shows the ability of the existing approaches to model each of the interoperability layers. In short, most of them covered the modeling of the field layer, which contains the physical components of the MG. Concerning the information/knowledge layer, the ontology-based approaches showed a potential information modeling, compared to the non-ontology based approaches, represented by the classification and the categorizing of the MG components, but lack in modeling the relationships between them. 
Table 1. Comparing existing power system information models regarding the interoperability aspect

\begin{tabular}{|l|c|c|c|}
\hline Features & Field Layer & $\begin{array}{c}\text { Information/Knowledge } \\
\text { Layer }\end{array}$ & Management Layer \\
\hline CIM [13] & $\checkmark$ & Partial & $\times$ \\
\hline NIST [19] & Partial & $\times$ & Partial \\
\hline OASIS [16] & $x$ & Partial & Partial \\
\hline MIRABEL [15] & Partial & Partial & $\times$ \\
\hline Prosumer [11] & Partial & Partial & $\times$ \\
\hline $\begin{array}{l}\text { Belgrade [10] } \\
\text { power }\end{array}$ & $\checkmark$ & Partial & $x$ \\
\hline $\begin{array}{l}\text { Upper ongineering } \\
\text { applications [14] }\end{array}$ & Partial & $x$ & \\
\hline
\end{tabular}

\subsubsection{Multi-objective aspects}

Table 2 summarizes the main commonalities and differences between existing approaches with respect to the five categories of aspects used in the achievement of the MG's objectives. In short, few took properly into account the identification aspect (Id), which consists of assigning a unique identifier to each component. In contrast, the operational aspect (Op) was the core of most of the existing models, whose aim was to standardize the technical vocabulary in the power systems, except in the MIRABEL system that mainly focuses on the energy market modeling. Clearly, as the comparison table shows, the economical aspect (Eco) was highly modeled through several major parameters designating operation and maintenance costs in the system (i.e., "StartCost" representing the startup cost of a device, "MainCost" representing the maintenance cost of a device, etc.). Moreover, the ecological aspect (Ecolo) was merely modeled through parameters related to the gas emissions of the components (i.e., CarbEss representing the carbon emission, EthylEss representing ethyl emission, etc.). However, the mobility aspect was almost absent in the existing information models, which represents the roles played by a component during its lifetime according to a certain context.

To sum up, none of the existing approaches covers all the identified aspects. In the following section, we provide a new information model OntoMG, aiming at resolving interoperability issues from the information perspective and integrate all the MGs' aspects related to meeting their objectives. 
Table 2. Comparing existing power system information models regarding the MG multi-aspect

\begin{tabular}{|l|c|c|c|c|c|}
\hline \multicolumn{1}{|r|}{ Features } & Id & Op & Mob & Eco & Ecolo \\
\hline Approaches & Partial & $\checkmark$ & $x$ & Partial & $x$ \\
\hline CIM [13] & Partial & $\checkmark$ & $x$ & $x$ & $x$ \\
\hline NIST [19] & $x$ & Partial & $x$ & $\checkmark$ & $\times$ \\
\hline OASIS [16] & Partial & $x$ & $x$ & $\checkmark$ & Partial \\
\hline MIRABEL [15] & $x$ & $\checkmark$ & $x$ & $x$ & Partial \\
\hline Prosumer [11] & Partial & $\checkmark$ & $x$ & $\checkmark$ & Partial \\
\hline Belgrade [10] & $x$ & $\checkmark$ & $x$ & $x$ & $x$ \\
\hline $\begin{array}{l}\text { Upper ontology for } \\
\text { power engineering } \\
\text { applications [14] }\end{array}$ & $x$ & & $x$ & \\
\hline
\end{tabular}

\section{Proposal}

In this paper, we introduce OntoMG, a full-fledged MG ontology-based information model that aims at resolving interoperability issues in a $\mathrm{MG}$ considering all its aspects/functionalities, namely: Identification, Operational, Mobility, Economical and Ecological. The information model is defined within the Information/Knowledge Layer. As mentioned previously, this research is undertaken in collaboration with Jema Irizar Group, leader of the ISare Microgrid project. The aim of ISare is to create a smart operable, reliable and efficient power system that enables the integration and the validation of the various renewable distributed generation systems and storage technologies. Before detailing OntoMG, we briefly describe our Microgrid management system architecture inspired from GridWise council's interoperability framework [9].

\subsection{An overview of the Microgrid Management System architecture}

Our MicroGrid Management System architecture is based on the GridWise council's interoperability framework [9], as presented in Fig. 3.

It consists of 3 main modules as follows:

- Field Layer (FL): it directly focuses on the digital exchange of data between the physical equipment of an MG and the establishment of a reliable low-level communication environment. This is achieved by using several standardized protocols such as BACnet [7] and Modbus [6] to transform communications to TCP/IP packets. Note that, the data exchanged between the components at this layer (voltage, frequency level, etc.) is stored in different data storage repositories depending on the technologies used. 


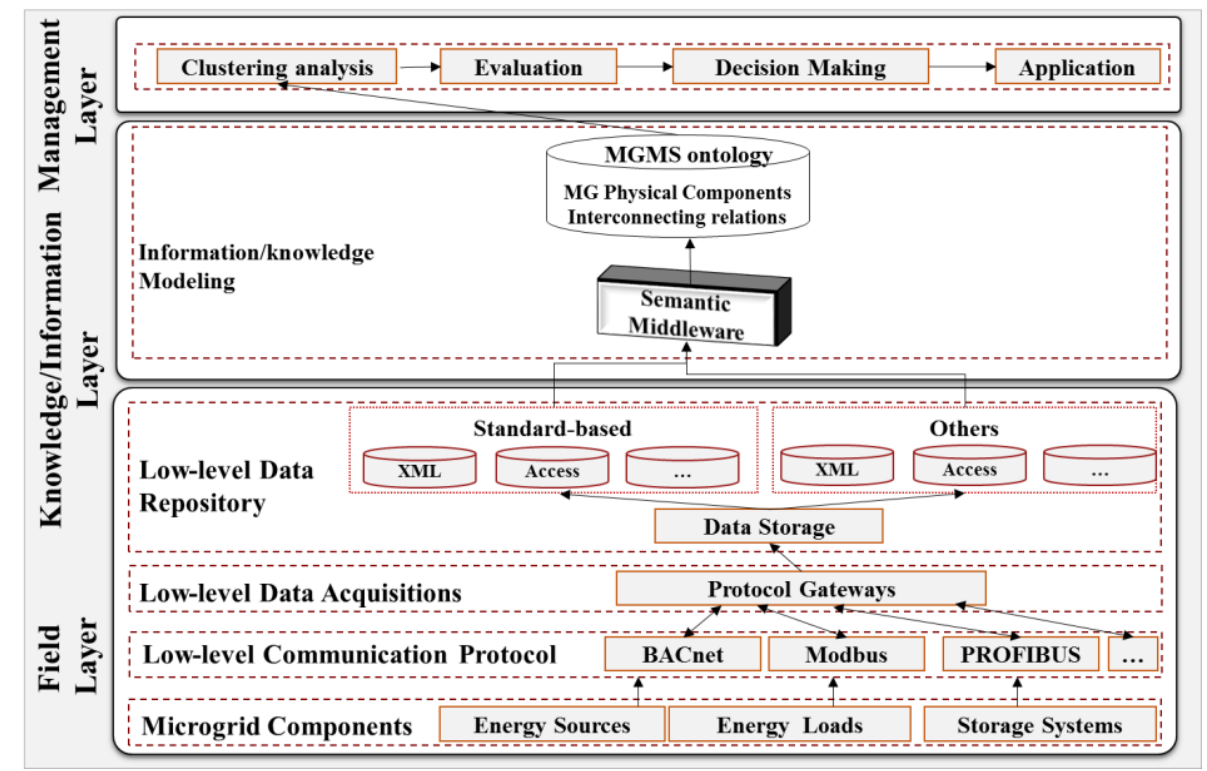

Fig. 3. MicroGrid information system architecture

- Information/Knowledge Layer (KIL): Since, it is not sufficient to understand the syntax or the grammar of the data exchanged to ensure a seamless communication between the components, it is primordial to capture its semantic meaning and to model it. Thus, this layer encompasses the semantic translation of the data coming from the low-level data repositories. The translated data plays an essential role in facilitating the interoperation and open up the possibility to model the new trends in today's energy systems (i.e., prosumers, power plants, electric vehicle, etc.).

- Management Layer (ML): it uses the information extracted from the KIL in order to provide advanced management and control services and functionalities. It consists of applying computational and optimization techniques, aiming to achieve the MG objectives (i.e., minimizing transmission losses, generating good power quality, minimization of green-house effect gases, etc.).

Since the communication within the FL is already established with the existence of standardized low-level communication protocols, thus, we provide here, a generic ontology-based model, aiming to model MG components, their parameters and additional aspects allowing the achievement of the MG's objectives.

\subsection{Why Ontology?}

In the recent years, the ontology gained a huge success in the representation of the domain specific knowledge and resolving interoperability issues [20]. Due to its various roles [21] in information systems and artificial intelligence, an ontology-based MG information model would provide a shared knowledge conceptualization allowing an easier system interaction and manipulation for non-computer scientists, while giving the system components reasoning capabilities and autonomy. 


\subsubsection{Ontology as a Shared Knowledge}

Since an MG consists of a number of heterogeneous components, it is primordial to define a shared representation of the exchanged information. In addition, each component has a direct/indirect impact on the other components and on the overall MG. Hence, it is necessary to have a shared collective representation allowing the study of the local and the global impact of each component in the power system. Furthermore, knowing that there are several existing energy system information models, an ontology would form the knowledge heart of the system, by providing enrichment and semantic expressiveness to the information but also allowing the description of specific situations, integration and alignment with multiple specific ontologies.

\subsubsection{Ontology as a Natural Language}

Since an MG is managed by non-computer-scientists, an ontology would help the users interact and manipulate the system in an easier way. Besides, an ontology would provide an organization that is flexible, and that naturally structures the information in multidimensional ways like finding more general/specific classes (e.g., a wind turbine concept is a type of the distributed energy sources). As well, an ontology would allow a more sophisticated data querying, for instance, finding information about "electricity sources that are DC sources but are neither fuel-cells nor photovoltaic".

\subsubsection{Ontology as a Reasoning Strategy}

Due to the intermittent aspect [22] of the renewable energy sources and the exposure of the MG to predictable and non-predictable events (MG anomalies, storms, etc.), an ontology modeling becomes essential since it can represent beliefs, goals, hypotheses, and predictions. These latter will give the components the ability to act and react autonomously or collectively according to a certain event. For instance, a diesel generator should stop working automatically when the renewable energy sources are able to satisfy all the loads power demands.

For all these reasons, we believe that modeling an MG using an ontology is a suitable choice allowing the resolving of the interoperability issues in a system consisting of numerous heterogeneous components, exposed to internal and external events.

\subsection{OntoMG Ontology}

Our ontology, called OntoMG, is a graph representing a collection of subject-relationobject triples, where:

- Nodes designate subjects, objects, or subject/object properties representing:

- MG branches and components (e.g., EnergyStorageBranch, WindTurbine, etc.)

- Corresponding property values (e.g., panelWidth, totalCost, etc.)

- Edges connecting source/destination nodes, designate relations representing:

- Relations between components (e.g., WindTurbine SubClassOf DistributedEnergySource, etc.),

- Property and value relations (e.g., windTurbine HasSpeed 50, solarPanel HasCost 7500 , etc.) 


\subsubsection{OntoMG Structure}

MGs encompass renewable and non-renewable generators, storage equipment and electrically connected loads. Moreover, they can be connected or isolated from the main grid. Our OntoMG structure is based on the common information model (CIM) proposed in [23] with several extensions to describe the aspects defined previously. The CIM is the core of the IEC 61970 standard, used to describe management information via a UML object oriented model.

In our model, a "Microgrid" class inherits from the "EnergyUnit", which allows the creation of several connected MG instances. Bringing in the branch concept defined in [11], an MG consists of three main branches, namely: 1) DESBranch (distributed energy source branch), 2) ESBranch (energy storage branch) and 3) ELBranch (energy load branch), where each branch has its own structure.

\subsubsection{OntoMG Multi-objective Aspects}

Our OntoMG aims to model all the aspects/functionalities participating in the achievement of the objectives of MG components and system. Hence, a suitable solution must cover the five aspects previously mentioned, namely: 1) identification (Id), 2) economical (Eco), 3) operation (Op), 4) mobility (Mob) and 5) ecological (Ecolo).

\subsubsection{Identification Aspect (Id)}

An MG consists of several heterogeneous devices, each having its own characteristics and operation modes during its lifetime. Thus, when joining an MG, each device is associated through OntoMG with an "identity" consisting of a number of parameters distinguishing it from the other devices and giving it the possibility to be automatically recognized. The three types of devices (the distributed energy sources, the energy storage systems, and the energy consumption loads) are sharing a number of identification parameters, consisting of the identifier (ID) which is a unique value, the device type (Type) where each type has its own characteristics, and the brand (Brand) of the device designating a certain provider.

In addition, each device has its own "power scheduler" constituting the device's signature (Sig), which is the power quantity produced, consumed/stored by the distributed generation device, the power consumption load, and the energy storage, respectively, at a certain time of date (TOD).

The identification aspect aims at giving a unique identity for each component. When this latter joins a new MG, it will be easier to recognize thanks to this identity, which facilitates its configuration and integration. Note that, the aggregation of all the MG components identities defines what we call the MG identity.

The ID aspect has several objectives, starting with the "dynamic power scheduling". Based on a similarity computation between the components signatures ( $\mathrm{ig}$ ), it is possible to propose an optimized schedule allowing to minimize the costs and the wasted power. For instance, considering two consumers $\mathrm{C} 1$ and $\mathrm{C} 2$, and despite owning the same consumption load (e.g., a washing machine), $\mathrm{C} 1$ has a higher consumption bill. Hence, the MG will be able, using the comparison between the two loads signatures, to propose a better schedule to $\mathrm{C} 1$. In addition, this aspect plays an essential role in the "MG configuration process". More specifically, since it is possible to recognize a new device, it will be easier to complete missing information and to extract implicit ones (i.e., 
type, brand, power consumption, etc.), which offers easier and faster component configurations.

\subsubsection{Economical Aspect (Eco)}

Due to the importance of the MG from an economic perspective, it is essential to model the economic parameters of the MG devices. Those parameters imply several features related to the components' participation in the Energy Market. Some of the main economic parameters of the device consist of the maintenance cost (MainCost), the total cost (Tot Cost), the startup cost (CostStart), the shutdown cost (CostStop) and the maintenance cost (MainCost).

The objective of the economical aspect is to manage the participation of the MG components in the energy market in order to reduce the power costs. In other words, it allows choosing the suitable device to be installed, based on its TotCost and MainCost. Once the devices are installed, the coststart and the Coststop play a main role in delegating the cheapest device that should be launched to satisfy a certain need in the power system.

\subsubsection{Operational Aspect (Op)}

The operational aspect encompasses the technical parameters related to the components operations during their lifetime in the MG. Since our model is based on the IEC 61850, this eases the exchanges of the technical information between the power system components.

The distributed energy source (DES) operation parameters package, encompasses the following parameters: the optimal efficiency (OpEff) expressed in \%, the nominal active power (NomAct $\mathrm{Pwr}$ ) in KW, and the operating status (Status) capturing if the device is working or not.

The energy storage (ES) parameters package, encompasses the following parameters: the energy storage lifetime ( $\mathrm{LifeTm}$ ) in "Years", the converter type (ConvTyp), the voltage ( $\mathrm{V} \circ \mathrm{lt}$ ) in $\mathrm{V}$, the storage device capacity (Cpty) in Ah, and the efficiency (Eff) in $\%$.

The energy load (EL) parameters package, encompasses the minimum power consumption (minCnsm) and the maximum power consumption (maxCnsm) expressed both in Watt.

The success key of an MG is its operation aspect, which is related to the components functioning during its lifetime in the system. Therefore, there is an essential need to model the technical information that defines the operations in the MG, to be able later to optimize the network operations (i.e., minimizing power losses, voltage variations, device loading, etc.). 


\subsubsection{Ecological Aspect (Ecolo)}

Knowing the importance of the MG in the integration of green energy production, it is essential to take into account the component's contribution in the environment. This participation is modeled using several parameters, mainly through the carbon emission ratio (CarbEss), the Ethylene emission ratio (EthylEss), and others gas emissions ratios, expressed in $\mathrm{g} / \mathrm{Kg}$. In addition, distributed energy resources are categorized according to the type of power that they are producing, whether it is a renewable energy source or not, using the parameter "DERCat".

Modeling the ecological aspect opens up the possibilities to make "green decisions" and consider various management strategies, by making the components participation in the environment visible. Thus, it is easier now through OntoMG to choose the cleaner energy source, able to satisfy the power needs.

\subsubsection{Mobility Aspect (Mob)}

Nowadays, there are already some devices that have the ability to move (i.e. electric vehicle, flywheels, etc.) during their lifetime within the same MG or to leave and join another one. In order to model this feature of those mobile components, a "geo" parameter was defined, which describes the location of the MG device using three possible values:

$$
\begin{aligned}
& \text { Geo }=\text { null: if the device cannot provide its location } \\
& \text { Geo }=g_{i} \quad: \text { if the device can provide its actual location } \\
& \text { Geo }=\left\{g_{1}, g_{2}, \ldots, g_{i}\right\}: \text { if the device can provide a location track log history }
\end{aligned}
$$

The objective of the mobility aspect is to optimize the MG cost efficiency. Due to device tracking, it will be easier to detect the location of any problem and fix it more rapidly. Besides, it is possible now to calculate which is the nearest available power source able to satisfy the load needs, and consequently reduce transmission losses. Besides, the Mob aspect emphasizes the components adaptability. In other words, a component will be able to take its preferences wherever it moves during its lifetime in the system. For instance, if an energy consumption load has a preference to be launched automatically at 7:00 every day while being in France, hence, it should be able to be launched at the same time during its displacement in Spain. Illustration

As mentioned previously, this research is undertaken in collaboration with Jema Irizar Group, leader of the ISare MG project. ISare MG is installed in San Sebastien - Spain and electrifies 12 offices. The generation system comprises $10 \mathrm{~kW}$ of PV, a nominal $53-\mathrm{kWh}$ battery bank, $105 \mathrm{~kW}$ of wind generation and a $120 \mathrm{~kW}$ diesel genset. A second PV array of about $15 \mathrm{~kW}$, mounted on the roof of the control system building, is connected to an SMA inverter and a 70-kWh of gas turbine to provide power for monitoring and communication. Recently, $50 \mathrm{~kW}$ of electric vehicle charger were installed, equipped with a protection system, to ensure a mobile power when needed.

\section{Illustration}

The aim of this study is to allow ISare MG project to create an interoperable Microgrid that enables the integration and the validation of the various new heterogeneous renewable distributed generation systems and various storage technologies. In addition 
and it is worthy to note that ISare MG is mainly managed by non-computer experts. Thus, developing a framework that enables an easier data querying and management was needed to help achieve the MG objectives.

Our ISare-OntoMG model, is an OWL graph, implemented on a central entity, requested by non-computer experts via a natural processing querying interface. This latter interprets grammatical and lexical units of a natural language expressed by the user, in order to form SPARQL queries. (This will be explained in a dedicated work.). SPARQL is a query language, that is, a semantic query language, able to retrieve and manipulate data stored in Web Ontology Language (OWL).

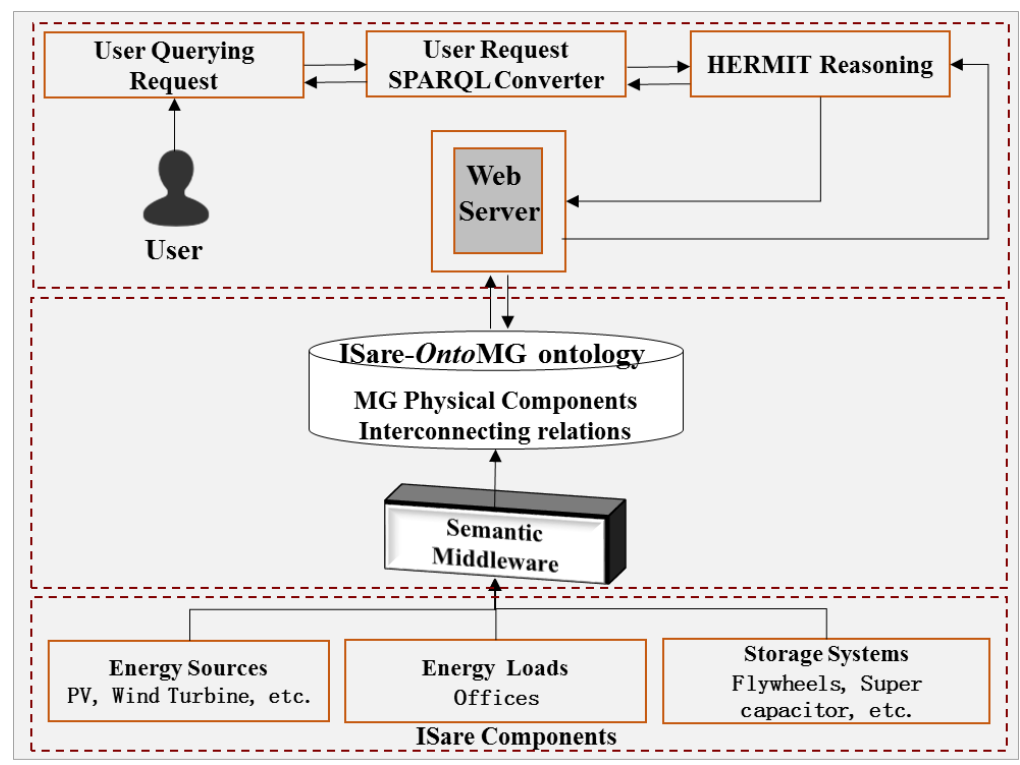

Fig. 4. ISare Framework Architecture

In the following, some of the advantages provided by our ISare-OntoMG are presented.

Starting with the identification aspect: ISare components were used to be identified using their brands and models only. This was restrictive to distinguish between components since several might have the same brand and model. With OntoMG, it is now possible to give each component a unique signature ( $\mathrm{Sig}$ ) representing the power produced/stored/consumed at a certain time of day.

Also OntoMG grants each component the mobility features, an important issue related to the high number of electric vehicles used in ISare, which was not possible in the existing information model. Henceforth, the MG manager is able now to track the moves of the some electric vehicles through a simple SPARQL query such as:

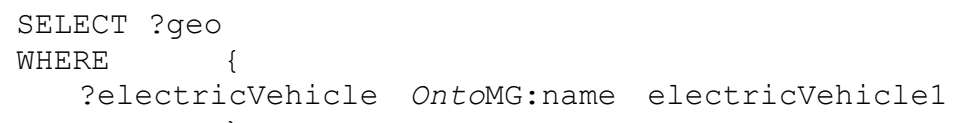


In the above query, the geo location of the electric vehicle having as name "electricVehicle1" is requested.

Concerning the ecological aspect, our model offers green strategies allowing to choose energy sources having the lowest gas emissions ratio, using a simple query such as:

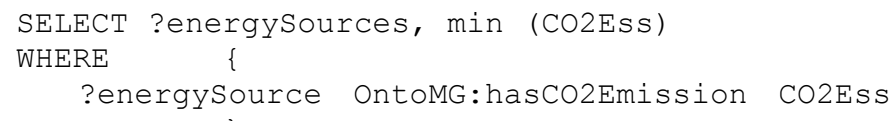

In the above query, all the energy sources having the lowest $\mathrm{CO} 2$ emission are requested.

\section{Conclusion}

This paper introduces OntoMG, an ontology-based information model that aims 1) to resolve interoperability issues encountered in the $\mathrm{MG}$ and 2) to achieve its functionalities and objectives (not fully covered in the existing information models). Our model is based on the CIM and the IEC 61850 standards, integrating 6 packages each related to a specific aspect involved in the achievement of the MG objectives, namely, 1) identification aspect (Id), related to the components unique identity in the system, 2) operation aspect (Op), related to the component's operating, 3) mobility (Mob) aspect related to the components displacements during their lifetime, 4) economical aspect (Eco), related to the components' participation in the Energy Market, 5) ecological aspect (Ecolo), related to the component's participation and effects in/on the environment.

An illustration was provided here through ISare project, a real MG build in the Basque Country, using our OntoMG model, highlighting the need to ensure a seamless communication between the MG components and integration of the new aspects to achieve the ISare objectives. Bearing in mind that modelling information is one of the major challenges in the MG management systems, one of our next steps consists of defining advanced management and control services and functionalities. This will be done by applying various optimization and scheduling techniques, aiming to achieve the MG objectives.

\section{References}

1. Fauzey, I.H.M., et al., Emergent Occupational Safety \&amp; Health and Environmental Issues of Demolition Work: Towards Public Environment. Procedia - Social and Behavioral Sciences, 2015. 168(0): p. 41-51.

2. $\quad \mathrm{Wu}, \mathrm{M}$., et al. Research on the architecture of Internet of things. in Advanced Computer Theory and Engineering (ICACTE), 2010 3rd International Conference on. 2010. IEEE. p.484-487.

3. Bouzid, A.M., et al., A survey on control of electric power distributed generation systems for microgrid applications. Renewable and Sustainable Energy Reviews, 2015. 44(0): p. 751-766.

4. Barnes, M., et al. Real-world microgrids-an overview. in System of Systems Engineering, 2007. SoSE'07. IEEE International Conference on. 2007. IEEE. p.1-8.

5. Ho, Q.-D. and T. Le-Ngoc, Chapter 5 - Smart Grid Communications Networks: Wireless Technologies, Protocols, Issues, and Standards1, in Handbook of Green Information and 
Communication Systems, M.S. Obaidat, A. Anpalagan, and I. Woungang, Editors. 2013, Academic Press. p. 115-146.

6.

Dao-gang, P., et al. Design and Realization of Modbus Protocol Based on Embedded Linux System. in Embedded Software and Systems Symposia, 2008. ICESS Symposia '08. International Conference on. 2008. p.275-280.

7. Gi Myung, K., et al. Design of a BACnet-ZigBee gateway for Smart Grid in buildings. in Conference Anthology, IEEE. 2013. p.1-5.

8. Bani-Ahmed, A., et al. Microgrid communications: State of the art and future trends. in Renewable Energy Research and Application (ICRERA), 2014 International Conference, IEEE, 2014. p.780-785.

9. Chassin, D.P. and L. Kiesling, Decentralized coordination through digital technology, dynamic pricing, and customer-driven control: The gridwise testbed demonstration project. The Electricity Journal, 2008. 21(8): p. 51-59.

10. Marko Bati, N.T., Dejan Paunovi, Sanja Vraneš, A NOVEL APPROACH TO MICROGRID DATA MODELLING.

11. Syed Gillani, F.L., Gauthier Picard, A Generic Ontology for Prosumer-Oriented Smart Grid. Workshop Pro-ceedings of the EDBT/ICDT 2014, 2014. p.134-139.

12. Buchmann, A. and B. Koldehofe, Complex event processing. it-Information Technology Methoden und innovative Anwendungen der Informatik und Informationstechnik, 2009. 51(5): p. 241-242.

13. McMorran, A.W., An introduction to iec 61970-301 \& 61968-11: The common information model. University of Strathclyde, 2007. 93: p. 124.

14. Catterson, V., et al., An upper ontology for power engineering applications, 2011. Available from: http://ewh.ieee.org/mu/pes-mas

15. Verhoosel, J., F.-J. Rumph, and M. Konsman. Modeling of Flexibility in Electricity Demand and Supply for Renewables Integration. in 2nd Workshop on eeBuildings Data Models, 27 October 2011, Sophia Antipolis, France. 2011.

16. Cox, W., D. Holmberg, and D. Sturek. Oasis collaborative energy standards, facilities, and zigbee smart energy. in Grid-Interop Forum. 2011.p.1-8.

17. Cox, W.T., T. Considine, and T. Principal, Architecturally significant interfaces for the smart grid. Grid-Interop-The road to an interoperable grid, Denver, Colorado, USA, 2009: p. 17-19.

18. Pagani, G.A. and M. Aiello, Service Orientation and the Smart Grid state and trends. Service Oriented Computing and Applications, 2012. 6(3): p. 267-282.

19. NIST Smart Grid Priority Action Plan 10: Standard Energy Usage Information. Available from: http://www.nist.gov/smartgrid/priority-actions.cfm.

20. Rui, D. and G. Deconinck. Multi-agent model and interoperability of a market mechanism of the Smart Grids. in Network Operations and Management Symposium Workshops (NOMS Wksps), 2010 IEEE/IFIP. 2010. p.312-315.

21. Gruber, T.R., Toward principles for the design of ontologies used for knowledge sharing? International journal of human-computer studies, 1995. 43(5): p. 907-928.

22. Kanoria, Y., et al. Distributed storage for intermittent energy sources: Control design and performance limits. in Communication, Control, and Computing (Allerton), 2011 49th Annual Allerton Conference. 2011. p.1310-1317.

23. Ming, D., Z. Zhengkai, and G. Xuefeng. CIM Extension of Microgrid Energy Management System. in Power and Energy Engineering Conference, 2009. APPEEC 2009. Asia-Pacific. 2009. p. 1-6. 